\title{
Variables asociadas a la carga en cuidadores primarios de personas con enfermedad renal crónica
}

\author{
Variables associated to burden in primary caregivers \\ of people with chronic kidney disease
}

\author{
Aranel de Jesús Arredondo Pantaleón y Samuel Jurado Cárdenas \\ Universidad Nacional Autónoma de México ${ }^{1}$
}

Autor para correspondencia: Aranel de J. Arredondo P., aruscamb@gmail.com.

\begin{abstract}
RESUMEN
El objetivo del presente estudio fue identificar las variables del cuidado que se relacionan significativamente con la carga del cuidador, y cuantificar la intensidad del malestar que producen dichas variables en los cuidadores primarios de personas con enfermedad renal crónica. Participaron 31 cuidadores, quienes respondieron la Escala de Carga del Cuidador de Zarit y un cuestionario elaborado ex profeso que evaluó la intensidad del malestar que generan las variables del cuidado; cabe mencionar que entre dichos cuidadores, $71 \%$ mostró algún grado de carga. Se encontraron relaciones significativas entre la carga y ciertas variables de tipo social, económico y hospitalario referidas a la rigurosidad del tratamiento, la salud del paciente, el rol del cuidador y la dependencia del paciente, mismas que también fueron valoradas por los participantes como generadoras de niveles elevados de malestar. Dado los resultados, es necesario identificar a los cuidadores con carga, al igual que diseñar e implementar intervenciones orientadas a promover su bienestar biopsicosocial.
\end{abstract}

Palabras clave: Cuidador primario; Carga del cuidador; Variables de cuidado; Enfermedad renal crónica.

\begin{abstract}
The objective of the present study was to identify care-related variables associated with caregiver's burden and quantify the intensity of discomfort produced by such variables in primary caregivers of chronic kidney disease patients. Thirty-one caregivers answered the Zarit Caregiver Burden Scale and a questionnaire evaluating the intensity of discomfort generated by the care variables. A total of $71 \%$ of caregivers showed some degree of burden. Significant relationships were found between the amount of burden and socioeconomic variables and with such hospital personnel practices such as the rigor of the treatment, the patient's health, the role of the caregiver, and the patient's degree of dependence. These variables also explained caregivers' distress and discomfort. These results point to the need to identify overwhelmed caregivers and design and implement interventions to promote their biopsychosocial well-being.
\end{abstract}

Key words: Primary caregiver; Burden; Care variables; Chronic kidney disease.

\footnotetext{
${ }^{1}$ Facultad de Psicología, Av. Universidad 3004, Col. Copilco Universidad, Coyoacán, 04510 Ciudad de México, México, correos electrónicos: aruscamb@gmail.com y jurado@servidor.unam.mx.
} 
Recibido: 03/06/2020

Aceptado: 05/09/2020

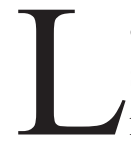

a enfermedad renal crónica (ERC en lo sucesivo) se define como un conjunto de anormalidades en la estructura y función de los riñones, las cuales persisten durante un periodo mayor a tres meses y tienen graves implicaciones en la salud (National Kidney Foundation, 2002).

Hoy día no se cuenta en México con un registro nacional que permita conocer con exactitud la cifra de casos de ERC en el país (Lastiri, 2016); sin embargo, de acuerdo con datos del Instituto Nacional de Estadística y Geografía (2019), durante el año 2018 la ERC fue la décima causa de muerte, con una cifra de 13,845 fallecidos. Asimismo, durante el año 2014 se posicionó como el padecimiento crónico más costoso para la Secretaría de Salud y el Instituto Mexicano del Seguro Social, lo que implica un considerable impacto financiero para dichas instituciones y las familias de quienes lo sufren (Figueroa, Gonzalez y Alarcon, 2016).

El esquema de tratamiento incluye la terapia sustitutiva de la función renal (diálisis peritoneal, hemodiálisis o trasplante), medicamentos, vacunas, dieta individualizada y control en la ingesta de líquidos (National Kidney Foundation, 2002). Lo anterior entraña diversas modificaciones en el estilo de vida que tienen un impacto físico, psicológico y social para quien padece la enfermedad como para su cuidador primario (CP en lo sucesivo), quien es la persona del entorno del paciente que asume la responsabilidad del mismo en un sentido amplio, que cubre sus necesidades y toma decisiones por y para él (Organización Mundial de la Salud, 1999).

El perfil general del CP reportado en la literatura es el de mujeres, adultas, cónyuges o hijas del paciente, amas de casa, casadas, con escolaridad básica (Aguilera et al., 2016; Laguado, 2019; Menati, Torabi, Andayeshgar y Khatony, 2020). En cuanto al número de horas dedicadas al cuidado se ha reportado que los cuidadores realizan su labor durante más de diez horas al día (Delgado, Barajas, Uriostegui y López, 2016; Romero, Bohórquez y Castro, 2018).
Entre las actividades del CP se encuentran las de brindar asistencia; apoyo social, emocional e instrumental al paciente; identificar y atender sus necesidades; manejar síntomas en el hogar; preparar los alimentos de acuerdo con la dieta renal; realizar la limpieza y el acondicionamiento del espacio físico; asumir los gastos; apoyar al paciente con las labores de higiene y vestido; verificar la toma de medicamentos, y transportar al receptor del cuidado (Avşar et al., 2015; Einollahi et al., 2009).

Como consecuencia de las demandas constantes de su labor, el CP suele reducir sus conductas de autocuidado y modificar sus hábitos alimenticios y de descanso, lo que puede originar alteraciones en su salud física. De acuerdo con la literatura, los problemas de salud que afectan en mayor medida a los CP son, a saber: complicaciones para caminar, dolor, fatiga, alteraciones estructurales en el cerebro, accidentes cerebrovasculares, diabetes, hipertensión y otras lesiones originadas al movilizar, cargar o transportar al paciente (Alnazly y Samara, 2014; Delgado et al., 2016; Haley, Roth, Howard y Safford, 2010; Mendoza et al., 2014; Smagula, Beach, Rosso, Newman y Schulz, 2017). El cuidado en casa se asocia con un aumento de las limitaciones funcionales de largo plazo (Caputo, Pavalko y Hardy, 2016). A su vez, en su estudio clásico, Schulz y Beach (1999) sostienen que el acto de cuidar es por sí mismo un factor de riesgo de mortalidad. De igual manera, se ha reportado que hay una asociación entre el número de horas dedicadas al cuidado y el riesgo de mortalidad del CP (Tseliou, Rosato, Maguire, Wright y O'Reilly, 2018).

Desde el punto de vista social, el CP encara circunstancias como la falta de tiempo para atender a otros miembros de su familia, escasas oportunidades para convivir con amigos y participar en actividades sociales o de ocio (Alnazly y Samara, 2014); asimismo, los CP con frecuencia refieren no contar con el apoyo de su familia, lo que deriva en conflictos en ese contexto. Lo anterior tiene como resultado el aislamiento social, los sentimientos de soledad y las dificultades para establecer relaciones interpersonales (Hoang, Green y Bonner, 2018). 
A los puntos anteriormente mencionados se suman los gastos económicos derivados del padecimiento, así como el hecho de que, como consecuencia de las constantes demandas que implica la enfermedad, muchos pacientes y cuidadores pierden su trabajo o deben renunciar a él, lo que conlleva a disponer de recursos insuficientes para cubrir tanto las necesidades del paciente como los requerimientos cotidianos de la familia, lo que se traduce en una gravosa carga financiera (Collins y Swartz, 2011; Surendran, Venugopal, Dongre y Paninjukunnath, 2018).

Las funciones del cuidador se asocian estrechamente al bienestar y a la estabilidad del paciente. En virtud de lo anterior, los CP perciben el cuidado como una labor en la cual existen estresores persistentes, incontrolables e impredecibles; luego entonces el cuidado cuidado cotidiano y permanente de una persona con ERC deriva en un acrecentamiento de la frustración y el cansancio del cuidador, lo que perjudica su salud psicológica (Collins y Swartz, 2011; Laguado, 2019; Surendran et al., 2018). Autores como Kang, Yu, Foo, Chan y Griva (2019) señalan que la labor de cuidar trae consigo una disminución de la calidad de vida y una reducción significativa de la salud psicológica; a su vez, Romero et al. (2018) midieron el grado de perturbación que el cuidado ocasiona en las diversas áreas de la vida del sujeto, siendo la psicológica el área más afectada.

De acuerdo con la literatura científica, la labor de cuidado produce diversas consecuencias en la esfera psicológica, tales como aflicción, tristeza, inseguridad, miedo, angustia, soledad, irritabilidad, desesperanza, resentimiento, culpa, depresión, ansiedad, estrés, insomnio y carga del cuidador (Avşar et al., 2015; Bardak et al., 2018; Bawazier, Stanley, Sianipar y Suhardjono, 2018; Caputo et al., 2016; Collins y Swartz, 2011; Hoang et al., 2018; Kang et al., 2019; Laguado, 2019; López, Ávila, Reyes y Miranda, 2018; Surendran et al., 2018; Tseliou et al., 2018; Velázquez y Espín, 2014).

La carga se refiere al grado de perturbación y malestar que la labor de cuidado ocasiona en las diversas áreas de la vida del sujeto, un estado que amenaza su salud integral (Zarit, Reever y Bach-Peterson, 1980). Tal obligación, a su vez, pue- de dividirse en objetiva y subjetiva. La primera se refiere a las actividades de cuidado y a las modificaciones que los CP experimentan en su vida cotidiana; mientras que la segunda alude a las preocupaciones, actitudes, emociones, percepciones y malestar subjetivo acerca del cuidado, así como el modo en que el CP vive la carga objetiva (Alvarado et al., 2011; Hoenig y Hamilton, 1966; Schene, Tessler y Gamache, 1994).

En la literatura especializada se encuentra ampliamente documentada la presencia de diversos niveles de carga en los CP de personas con ERC. Autores como Rivero, Segura y Juárez (2015), Delgado et al. (2016), Hoang et al. (2018) y Menati et al. (2020) reportan niveles que van de moderados a intensos. Usman Shah et al. (2017) y Cubas, Sánchez y Gálvez (2019) hallaron en sus estudios que dicha carga se ubicaba entre los niveles de leve a moderado.

Distintos autores han documentado variables relevantes relacionadas con el cuidador y con las características del cuidado que producen malestar y carga en los CP de personas que viven con ERC, entre las que se encuentran: edad del CP y del paciente, factores económicos y laborales, tipo de tratamiento, apoyo percibido, ser o no cuidador único, responsabilidad en el cuidado y el tratamiento, tiempo de cuidado del paciente desde el momento del diagnóstico, salud del $\mathrm{CP}$, número de horas dedicadas al cuidado, permanencia de la persona en diálisis y cuidado de sí mismo (Bardak et al., 2018; Cubas et al., 2019; Denardi et al., 2013; Díaz, Ramones, Vargas y Alejo, 2018; Franco y Romero, 2019; Laguado, 2019; Usman Shah et al., 2017; Velázquez y Espín, 2014).

De igual manera, se ha demostrado que las variables propias de las condiciones del cuidado también inciden en el desarrollo de la carga. Entre dichas variables se encuentran la frecuencia, intensidad y complejidad del tratamiento; el seguimiento riguroso de los procedimientos; las dudas, conocimientos y capacitación del CP sobre la enfermedad; los cuidados y el tratamiento; las urgencias inesperadas; las complicaciones y episodios agudos de la enfermedad; los reingresos hospitalarios y la duración de estos; el grado de dependencia del receptor del cuidado; la solicitud por parte del paciente de más ayuda de la que real- 
mente necesita; la modificación de las rutinas y horarios; la disminución del tiempo libre; los problemas para desplazarse desde el hogar al hospital; la cronicidad del padecimiento; la progresión de la enfermedad; la inestabilidad en la salud del paciente y la posibilidad de que fallezca (Aguilera et al., 2016; Cervantes et al., 2020; Cubas et al., 2019; Denardi et al., 2013; Díaz et al., 2018; Hoang et al., 2018; Laguado, 2019; López et al., 2018; Rivero et al., 2015; Surendran et al., 2018; Usman Shah et al., 2017).

Las variables mencionadas ponen de manifiesto el impacto del cuidado en las diferentes áreas de la vida de la persona encargada de desempeñar dicha labor. Si bien es cierto que las variables que se relacionan con el malestar y el desarrollo de carga en el CP se han documentado ampliamente, son escasos los estudios en los que se cuantifica la percepción del malestar que cada una de las variables del cuidado genera en los cuidadores.

Derivado de las anteriores consideraciones, el objetivo general del presente estudio fue identificar las variables del cuidado que se relacionan significativamente con la carga del cuidador mediante el análisis de la relación entre los puntajes obtenidos en la Escala de Carga de Zarit y los alcanzados en las variables incluidas en un cuestionario elaborado ex profeso para la presente investigación, así como cuantificar la intensidad del malestar subjetivo que producen dichas variables en los cuidadores primarios de personas con ERC. Como objetivos específicos se establecieron los siguientes: describir el perfil sociodemográfico de los CP de personas con ERC, identificar los niveles de carga del cuidador y detectar las necesidades de intervención psicológica de los CP que acudían al servicio de nefrología del Hospital General de Iztapalapa "Dr. Juan Ramón de la Fuente".

\section{MÉTODO}

\section{Participantes}

Se llevó a cabo un estudio observacional, correlacional y de alcance descriptivo en el que colaboraron 31 cuidadores primarios de pacientes con enfermedad renal crónica, seleccionados median- te la técnica de muestreo intencional no probabilístico. El número de cuidadores que participaron en el presente estudio equivale a $73.8 \%$ del total de pacientes inscritos en alguna modalidad de tratamiento sustitutivo del servicio de nefrología de la institución.

Los criterios de inclusión fueron el que manifestaran verbalmente ante los investigadores ser los cuidadores primarios, ser mayores de edad, acceder a participar de manera voluntaria en el estudio, aceptar firmar el formato de consentimiento informado y no estar recibiendo algún tipo de tratamiento psicológico o psiquiátrico.

\section{Instrumentos}

\section{Formato de consentimiento informado.}

Dicho formato fue redactado con base en los lineamientos de la Comisión Nacional de Bioética y la Guía Nacional para la Integración y el Funcionamiento de los Comités de Ética en Investigación, y autorizado por la Secretaría de Salud de la Ciudad de México. En la primera parte de este instrumento se proporciona información sobre la investigación y las consideraciones éticas de la misma, mientras que en la segunda sección los cuidadores acceden a participar firmando el citado formato.

\section{Entrevista semiestructurada.}

Esta entrevista incluye dieciocho preguntas organizadas de la siguiente manera: diez recaban información sobre los datos sociodemográficos; siete se relacionan con las características del cuidado, tales como el tipo de tratamiento que recibe el paciente, el tiempo durante el cual se ha ejercido el rol de cuidador, el número de horas al día dedicadas, y de ser el caso, el número de otras personas cuidadas además del paciente, enfermedades del cuidador y tratamiento que recibe en caso de ser así; finalmente, con el objetivo de identificar las necesidades de intervención, se incluyó la siguiente pregunta: "Si se le ofreciera un tratamiento psicológico, ¿qué temas le gustaría abordar?”.

Escala de Carga del Cuidador de Zarit (Zarit, Eiler y Hassinger, 1985).

Se utilizó la validación realizada en México por Montero, Jurado, Valencia, Méndez y Mora (2014). 
Se trata de una escala autoaplicable, integrada por 12 reactivos con cinco opciones de respuesta en una escala tipo Likert (de "Nunca" a "Siempre"). Los ítems se agrupan en tres factores que explican $50 \%$ de la varianza: impacto del cuidado, relación interpersonal y expectativas de autoeficacia. Cuenta con un coeficiente alfa de Cronbach de .84, con un modelo que cuenta con un buen ajuste, con valores iguales o superiores a .90. La escala se califica sumando las puntuaciones de todos los reactivos. El nivel de carga se interpreta de la siguiente manera: de 12 a 22 puntos: ausencia de carga; de 23 a 29 puntos: carga leve, y de 30 a 60 puntos: carga excesiva.

\section{Cuestionario elaborado ex profeso para el estudio.}

Se utilizó este instrumento para medir la intensidad del malestar que producen las variables relativas al cuidado en los CP. Está conformado por 31 reactivos con ejemplos de condiciones relacionadas con el cuidado de pacientes con ERC (por ejemplo, acompañarlo a la sesión de hemodiálisis). Los reactivos del cuestionario están organizados en las siguientes áreas: apoyo social, rigurosidad del tratamiento, aspectos financieros, modificaciones en la vida cotidiana, periodicidad de las visitas al hospital, hospitalización, estado de salud y pronóstico del paciente, rol del cuidador, dependencia del paciente y conocimientos sobre el tratamiento y necesidad de información. El formato de respuesta de los reactivos se presenta en una escala tipo Likert que va de 0 a 5 , en la cual debe puntuarse la intensidad del malestar que genera cada una de las condiciones que abordan los ítems, en donde 0 indica "No me genera malestar", 5 equivale a "Me genera un extremo malestar" y NA "No se aplica a mi situación".

Dicho cuestionario se construyó con base en la revisión de la literatura acerca de los aspectos del cuidado que se relacionan con el desarrollo de carga en los cuidadores primarios de personas con ERC, así como en las características de la enfermedad y los componentes del tratamiento. El instrumento se sometió a un proceso de validación por juicio de expertos en el que participaron dos médicos especialistas en nefrología y trasplante, así como una psicóloga especialista en psicología de la salud, quienes consideraron que el cuestiona-

Instituto de Investigaciones Psicológicas - Universidad Veracruzana rio era claro, pertinente y apto para aplicarse en la población a la cual se dirigía. Asimismo, se piloteó en la comunidad con 20 cuidadores primarios de personas con ERC para determinar la claridad y pertinencia de los reactivos y la facilidad para responder el cuestionario, sin que los participantes reportaran haber tenido dificultades para contestarlo.

\section{Procedimiento}

El estudio se sometió a la evaluación por parte del Comité de Ética en Investigación de la Secretaría de Salud de la Ciudad de México. Una vez obtenido el dictamen aprobatorio por parte de dicha institución, así como la carta de no inconveniencia por parte del Hospital General de Iztapalapa "Dr. Juan Ramón de la Fuente", se acudió a la sala de espera del servicio de nefrología del hospital, donde la autora principal invitó a las personas identificadas como cuidadores primarios a participar en el estudio, explicando sus objetivos e importancia y en qué consistía su participación. A quienes accedieron y cumplieron con criterios de inclusión, se les leyó el consentimiento informado y se les solicitó firmarlo, reiterándoles las consideraciones éticas del estudio. Se les proporcionaron los instrumentos y se les explicó la manera de llenarlos, indicándoles que en caso de existir alguna duda, podían preguntar lo que desearan. Al terminar, se agradeció su colaboración.

\section{RESULTADOS}

El análisis de datos se llevó a cabo utilizando el programa estadístico SPSS, v. 20, de Windows. Con el objetivo de identificar las variables de cuidado vinculadas significativamente con la carga del cuidador, se analizó la relación entre los puntajes obtenidos en la Escala de Carga del Cuidador de Zarit y las puntaciones obtenidas en cada una de las variables del cuidado incluidas en el cuestionario elaborado ex profeso para la investigación. Para ello, se empleó el estadístico no paramétrico rho de Spearman. Los datos obtenidos muestran que 18 de las 31 variables incluidas en el cuestionario guardan una relación estadísticamente significativa 
con la carga del cuidador. Dichas variables corresponden a nueve de las diez áreas ya mencionadas en la descripción del cuestionario. Las correlaciones fueron de bajas a moderadas. Los resultados obtenidos en el coeficiente rho de Spearman y el nivel de significancia de cada una de las variables se muestran en la Tabla 1.

Tabla 1. Variables del cuidado que muestran una relación estadísticamente significativa con la carga del cuidador y medias de malestar subjetivo generado por dichas variables.

\begin{tabular}{|c|c|c|c|c|}
\hline ÁREA & VARIABLE & $\begin{array}{c}\text { rho } \\
\text { de Spearman }\end{array}$ & Sig. & Media \\
\hline Apoyo social & No contar con ayuda para cuidar al paciente. & .37 & .04 & 3.29 \\
\hline \multirow{3}{*}{$\begin{array}{l}\text { Rigurosidad } \\
\text { del tratamiento }\end{array}$} & Seguir correctamente las instrucciones de los tratamientos. & .57 & .00 & 3.74 \\
\hline & Planificar los alimentos de acuerdo a la dieta. & .48 & .01 & 3.29 \\
\hline & $\begin{array}{l}\text { Posibilidad de que el paciente no siga la dieta mientras } \\
\text { el cuidador no está presente. }\end{array}$ & .37 & .04 & 3.16 \\
\hline Aspectos financieros & Gastos económicos relacionados con la enfermedad. & .57 & .00 & 4.84 \\
\hline \multirow{4}{*}{$\begin{array}{l}\text { Modificaciones } \\
\text { en la vida cotidiana }\end{array}$} & Modificación de los horarios de comidas. & .67 & .00 & 3.03 \\
\hline & Reducción del tiempo libre. & .63 & .00 & 3.42 \\
\hline & Modificación de los horarios de sueño. & .56 & .00 & 3.26 \\
\hline & $\begin{array}{l}\text { Modificación de las rutinas para adaptarlas a las necesidades } \\
\text { del paciente. }\end{array}$ & .49 & .01 & 3.03 \\
\hline \multirow{3}{*}{$\begin{array}{l}\text { Periodicidad } \\
\text { de las visitas al hospital }\end{array}$} & $\begin{array}{l}\text { Horas de espera en el hospital mientras el paciente está en sesión } \\
\text { de hemodiálisis. }\end{array}$ & .62 & .00 & 2.84 \\
\hline & $\begin{array}{l}\text { Tiempo invertido en acompañar al paciente a las sesiones } \\
\text { de hemodiálisis. }\end{array}$ & .60 & .00 & 3.10 \\
\hline & Acompañar al familiar a consulta. & .50 & .01 & 3.65 \\
\hline \multirow{2}{*}{ Hospitalización } & Cuidar al paciente durante la hospitalización. & .61 & .00 & 3.52 \\
\hline & Condiciones adversas del CP durante la hospitalización. & .59 & .00 & 3.48 \\
\hline \multirow{2}{*}{$\begin{array}{l}\text { Estado de salud } \\
\text { y pronóstico del paciente }\end{array}$} & $\begin{array}{l}\text { Posibilidad de fallecimiento del paciente debido a la ERC } \\
\text { o comorbilidades asociadas. }\end{array}$ & .45 & .01 & 4.55 \\
\hline & $\begin{array}{l}\text { Posibilidad de recibir malas noticias sobre la salud del paciente } \\
\text { durante la consulta o sesión de tratamiento. }\end{array}$ & .37 & .00 & 4.45 \\
\hline Rol del cuidador & Ser ella/él quien se hace responsable del paciente. & .50 & .00 & 4.13 \\
\hline Dependencia del paciente & El paciente solicita más ayuda de la que realmente necesita. & .44 & .01 & 2.74 \\
\hline
\end{tabular}

Las correlaciones son significativas al .05

Una vez identificadas las variables de cuidado que mostraron una relación significativa con la carga, se calcularon las medias de las mismas para cuantificar la intensidad del malestar subjetivo que producen, encontrándose que las cuatro que obtuvieron mayores puntuaciones como generadoras de malestar en los cuidadores fueron los gastos económicos relacionados con la enfermedad, la posibilidad de que el paciente falleciera debido a la ERC o a comorbilidades asociadas, la posibilidad de recibir malas noticias sobre el estado de salud del paciente cuando acudían a las citas médicas o a las sesiones de tratamiento, y ser la persona que se hace responsable del paciente.
Se efectuaron análisis descriptivos para describir el perfil sociodemográfico de los participantes. De acuerdo con los datos obtenidos, en su mayoría los cuidadores fueron del sexo femenino $(80.6 \%)$, amas de casa (54.8\%), casadas $(74.2 \%)$, con escolaridad de primaria $(38.7 \%)$ y religión católica $(90.3 \%)$. La media de edad fue 51.16 años (ubicándose en un rango de edad de 19 a 79 años). Respecto al parentesco, predominaron los cónyuges del paciente $(38.7 \%)$, aunque también fue elevada la presencia de hijas e hijos $(22.6 \%)$. Todos los participantes residían en la Ciudad de México.

En cuanto a las características de la labor de cuidado y la salud del CP, se encontró que $64.5 \%$ 
de los participantes eran cuidadores de personas en tratamiento de hemodiálisis, habían desempeñado el cuidado durante un promedio de tres años con tres meses y dedicaban una media de 15.26 horas al día a su labor de cuidar. Respecto a la presencia de otras personas a cargo del cuidador, $80.6 \%$ de los participantes refirió cuidar únicamente al paciente con ERC. En lo referente a la salud de los CP, $35.5 \%$ de ellos refirió padecer alguna enfermedad, siendo la diabetes mellitus la más frecuente $(n=7)$. De los cuidadores que reportaron algún padecimiento, 90.9\% afirmó recibir tratamiento.

Asimismo, se realizó una correlación rho de Spearman para establecer la relación entre la carga y las siguientes características del cuidado: edad del cuidador, tiempo durante el cual ha ejercido el cuidado y número de horas al día dedicadas a su labor. No se hallaron relaciones estadísticamente significativas entre dichas variables (edad del cuidador: $r=.03, p=.86$; tiempo que ha ejercido el cuidado: $r=.01, p=.97$, y horas al día dedicadas a su labor: $r=.01, p=.94$ ).

Con el fin de conocer si había diferencias estadísticamente significativas en la carga dependiendo del tipo de tratamiento que recibía el paciente, se utilizó el estadístico no paramétrico de comparación U de Mann-Whitney. No se encontraron diferencias estadísticamente significativas entre las medias de carga entre los CP de personas en diálisis y hemodiálisis $(\mathrm{Z}=-0.95, \mathrm{U}=87, p=.34)$.

Los datos obtenidos en los análisis descriptivos concernientes a los puntajes en la Escala de Carga del Cuidador de Zarit indican que $42 \%$ de los CP mostraba una carga intensa, mientras que $29 \%$ registró una carga leve, lo que dio como resultado un porcentaje acumulado de .71 de cuidadores con algún grado de carga.

Por último, el análisis del reactivo correspondiente a las necesidades de apoyo psicológico por parte del cuidador arrojó los siguientes resultados: $20 \%$ de los participantes externó requerir estrategias para manejar la ansiedad, el estrés y la depresión; 20\% solicitó estrategias para afrontar óptimamente los retos y dificultades relacionados con el cuidado; $14.3 \%$ consideró importante aprender a obtener apoyo social y mejorar la comunicación con su familia y el paciente, y 11.4\% manifestó necesitar aprender a cuidarse mejor.

\section{DISCUSIÓN}

El objetivo general del presente estudio fue determinar la relación entre la carga y las variables de cuidado mediante el análisis de la relación entre los puntajes en la Escala de Carga del Cuidador de Zarit y los obtenidos en las variables de cuidado en el cuestionario elaborado ex profeso para la investigación, así como cuantificar la intensidad del malestar que dichas variables producen en los cuidadores primarios que asistían a pacientes con ERC. En general, los hallazgos son congruentes con los reportados en la literatura. Estudios previos han apuntado variables que en este trabajo también resultaron tener una asociación significativa con la carga y producir malestar subjetivo, tales como la disminución del tiempo libre, la solicitud por parte del paciente de más ayuda de la que realmente necesita, ser el cuidador quien se hace cargo de la persona con ERC, seguir correctamente las instrucciones de los tratamientos, modificar las rutinas para adaptarlas a las necesidades del receptor del cuidado, modificar los horarios, no contar con ayuda para cuidar al paciente y apreciar la posibilidad de que el receptor del cuidado falleciera por la enfermedad que padece o por comorbilidades asociadas a la misma, lo cual coincide con la evidencia previa acerca de la carga relacionada con las alteraciones en la vida cotidiana, las demandas derivadas de la enfermedad y el grado de dependencia del paciente, así como la importancia del apoyo social en el desempeño de su labor (Aguilera et al., 2016; Cervantes et al., 2020; Cubas et al., 2019; Denardi et al., 2013; Díaz et al., 2018; Hoang et al., 2018; Surendran et al., 2018; Usman Shah et al., 2017; Velázquez y Espín, 2014).

Asimismo, un resultado consistente con estudios previos fue el de la variable relacionada con los gastos económicos derivados de la enfermedad (Cubas et a1., 2019; Laguado, 2019; Surendran et al., 2018), la que, además de relacionarse significativamente con la carga, obtuvo la media mayor 
de malestar subjetivo. Se trata de un área que merece una mención aparte por el impacto social que produce en razón del carácter de la ERC como un problema de salud pública asociado a altos costos económicos para los servicios de salud, mismos que se traducen en gastos elevados para el paciente y su familia (Figueroa et al., 2016). Los egresos que genera el padecimiento no se limitan a costear el tratamiento sustitutivo, sino que también abarcan componentes como la dieta, los medicamentos y los gastos del traslado periódico al hospital para acudir a las sesiones de tratamiento, estudios y consultas. A lo anterior se agregan los gastos familiares cotidianos, y el hecho de que frecuentemente los pacientes y los cuidadores pierden su trabajo como resultado de las demandas que impone la enfermedad, lo que reduce las fuentes de ingresos y se convierte en un estresor significativo que se asocia con la carga en el cuidador.

En cuanto a las variables relacionadas con la alimentación, se encontró que planificar los alimentos de acuerdo a la dieta y la posibilidad de que el paciente no siga el régimen alimenticio mientras el cuidador no se encuentra presente, supervisándolo, son condiciones del cuidado que se relacionan de manera significativa con la carga. Lo anterior adquiere sentido dado que la alimentación y la adherencia al régimen nutricional prescrito son pilares fundamentales para el control de la ERC. No cumplir con la dieta genera efectos nocivos en la salud del paciente, tales como malnutrición, enfermedades cardiovasculares, proteinuria e incremento en la tensión arterial (Pérez, González, López, Sánchez y Selgas, 2017). La preparación de los alimentos corre en gran medida a cargo del cuidador, por lo que las complicaciones derivadas de no seguir las indicaciones nutricionales pueden implicar además, una responsabilidad social para quien ejerce el cuidado, quien generalmente espera satisfacer a cabalidad las expectativas sobre su labor.

En lo que concierne a la periodicidad de las visitas a la institución de salud, dos de las variables en las que se halló una asociación estadísticamente significativa con la carga fueron el tiempo invertido en acompañar al paciente a su sesión de hemodiálisis, así como las horas de espera en el hospital mientras recibe el tratamiento. Lo ante- rior no solo tiene implicaciones relacionadas con la modificación de las rutinas, sino que además puede estar vinculado al hecho de que las sesiones de hemodiálisis tienen una duración promedio de tres a cuatro horas, tiempo durante el cual el cuidador debe permanecer en la sala de espera no únicamente aguardando la salida de su paciente, sino para estar disponible en caso de que se presente alguna complicación durante la sesión. Lo anterior puede representar una labor tediosa y constituir un estresor significativo, toda vez que existe la expectativa de que cada sesión transcurra sin eventualidades.

Acompañar al paciente a las consultas es una variable en la que pueden estar implicados factores como el tiempo, los ajustes que el cuidador debe hacer a sus rutinas, los sentimientos de incertidumbre y emociones como el temor y la preocupación, dado que otra variable que mostró una relación estadísticamente significativa con la carga, siendo una de consideradas como generadoras de mayor malestar, fue la posibilidad de recibir malas noticias sobre la salud del paciente durante la consulta o la sesión de tratamiento, lo cual puede implicar que las citas se perciban como una situación que de suyo implica la ocurrencia de diversos estímulos aversivos y estresores significativos, al igual que la producción de ideas y emociones anticipadas relacionadas con la progresión de la enfermedad y el futuro del paciente.

Respecto a las variables de la hospitalización, las que mostraron estar asociadas con la carga fueron las de cuidar al paciente durante la estancia intrahospitalaria, como las condiciones en las que se halla el CP durante la hospitalización del receptor del cuidado. Durante la práctica clínica en los servicios de nefrología puede observarse que, adicionalmente a la incertidumbre, el temor, el estrés, los gastos económicos y la fatiga que puede generar una hospitalización, los CP también se enfrentan a condiciones desfavorables, como incomodidad, falta de espacio físico y privacidad, ausencia de condiciones para asearse, descansar o alimentarse, restricciones para comunicarse con el resto de la familia y acompañamiento del paciente en los estudios y procedimientos, lo que aumenta su malestar físico y psicológico, en una situación que, de acuerdo con autores como Cervantes et al. 
(2020) y Rivero et al. (2015), es percibida en sí misma como adversa.

En cuanto al perfil sociodemográfico de los $\mathrm{CP}$, los hallazgos del presente estudio coinciden con los reportados en la literatura especializada, en los que predominan los cuidadores del sexo femenino, los cónyuges del paciente, casados en su mayoría, las amas de casa y una baja escolaridad (Aguilera et al., 2016; Laguado, 2019; Menati et al., 2020).

Por lo que respecta al número de horas dedicadas al cuidado, los datos concuerdan con los resultados reportados por Delgado et al. (2016) y Romero et al. (2018), quienes encontraron que los CP gastaban más de diez horas al día al cuidado de su paciente; de manera similar, los participantes de esta investigación pasaban en promedio 15.26 horas diarias ejerciendo esa labor. Pese a ello, el número de horas dedicadas al cuidado, el tiempo durante el cual se ha ejercido el cuidado y la edad del cuidador fueron variables que no mostraron tener una asociación estadísticamente significativa con la carga, a diferencia de los reportados por Laguado (2019) y Usman Shah et al. (2017).

Con relación al tipo de tratamiento, en los datos de este trabajo no se hallaron diferencias estadísticamente significativas entre la carga de los CP en hemodiálisis y diálisis, lo que concuerda con los de Aguilera et al. (2016), quienes no encontraron diferencias significativas entre los cuidadores de pacientes en ambos tratamientos.

En el caso de la salud física de los cuidadores, más de un tercio refirió padecer alguna enfermedad, siendo la diabetes mellitus la enfermedad más frecuente, lo que concuerda con los resultados de Delgado et al. (2016), y de ellos, 90.9\% afirmó recibir tratamiento. En cuanto al porcentaje de $\mathrm{CP}$ que padecían alguna enfermedad y recibían atención médica, Cervantes et al. (2020) apuntan que los cuidadores de personas con ERC, después de ser testigos de la enfermedad del paciente, tratan de mejorar sus hábitos de autocuidado para no enfermar debido a su experiencia. Sin embargo, dados los niveles de carga y la relación significativa con variables como la reducción del tiempo libre, la modificación de las rutinas y el tiempo invertido en acompañar al paciente al tratamiento, debe considerarse la posibilidad de que algunos de los cuidadores no dispongan del tiempo suficiente para su autocuidado, y que al priorizar la enfermedad del paciente no acudan a revisiones periódicas $\mathrm{y}$, por ende, desconozcan si han desarrollado algún padecimiento.

En lo que se refiere a los niveles de carga del cuidador, 71\% mostró algún grado de esta, hallazgo que apoya los resultados de Romero et al. (2018), quienes encontraron que el área psicológica era la más afectada al desempeñar la labor de cuidar, y de igual modo son congruentes con la literatura que reporta niveles de moderados a intensos (Delgado et al., 2016; Hoang et al., 2018; Menati et al., 2020; Rivero et al., 2015).

Por último, las necesidades de apoyo psicológico son coincidentes con las principales áreas afectadas por la labor de cuidado reportadas en la literatura, lo que, junto al porcentaje de carga encontrado y los datos concernientes a las variables de cuidado relacionadas con aquella, ponen de manifiesto la necesidad de incluir al CP dentro del esquema integral de atención al paciente con ERC. Autores como Rivero et al. (2015), Bardak et al. (2018) y Kang et al. (2019) subrayan la importancia de evaluar periódicamente el estado de los cuidadores, implementar intervenciones psicológicas y educativas para ellos, así como promover el trabajo interdisciplinario en aras de favorecer su bienestar biopsicosocial, además de fomentar la institucionalización de su labor impulsando la creación de espacios para su capacitación, el cuidado de su salud y el fortalecimiento de sus redes de apoyo, lo que al mismo tiempo se traducirá en un beneficio para los pacientes.

Desde este punto de vista, la intervención del psicólogo entrenado en el campo de la salud cobra especial relevancia en razón de que es el especialista en la evaluación y el manejo de los aspectos conductuales, cognitivos y emocionales de la enfermedad, quien puede diseñar y poner en práctica intervenciones cuya eficacia y efectividad se sustenten en la evidencia.

Finalmente, la principal limitación del presente estudio fue el tamaño de la muestra, lo que no permite generalizar los resultados.

Cabe mencionar que en el servicio donde se llevó a cabo la investigación no hay pacientes en protocolos de trasplante ni de postrasplante, lo 
cual no permitió conocer las variables asociadas a la carga en sus cuidadores, por lo que se sugiere que en futuros estudios se incluya a CP que asistan a dicha población. Por último, se sugiere que en trabajos posteriores sean consideradas la ansiedad y la depresión, así como las alteraciones del sueño, con la finalidad de identificar el grado de relación que guardan con los niveles de carga.

\section{AGRADECIMIENTOS}

El presente estudio se deriva de la tesis doctoral del primer autor bajo la dirección del segundo. Los autores agradecen al Consejo Nacional de Ciencia y Tecnología (CONACYT), que otorgó la beca número 449061, con número de CVU 363862; asimismo, al Comité de Ética en Investigación de la Secretaría de Salud de la Ciudad de México, al Hospital General de Iztapalapa “Dr. Juan Ramón de la Fuente” por las facilidades otorgadas para llevar a cabo esta investigación, y a los participantes del estudio.

Citación: Arredondo P., A.J. y Jurado C., S. (2022). Variables asociadas a la carga en cuidadores primarios de personas con enfermedad renal crónica. Psicología y Salud, 32(1), 5-16. https://doi.org/10.25009/pys.v32i1.2706.

\section{REFERENCIAS}

Aguilera, F.A., Castrillo, C.E., Linares, B., Carnero, G.R., Alonso, R.A., López, R.P. y Prieto, V.M. (2016). Análisis del perfil y la sobrecarga del cuidador de pacientes en Diálisis Peritoneal y Hemodiálisis. Enfermería Nefrológica, 19(4), 359-365. http://dx.doi.org/10.4321/S2254-28842016000400007.

Alnazly, E. y Samara, N. (2014). The Burdens on Caregivers of Patients above 65 Years Old Receiving Hemodialysis: A Qualitative Study. Health Care: Current Reviews, 2(1), 1-6. http://doi.org/10.4172/2375-4273.1000118.

Alvarado, R., Oyanedel, X., Aliste, F., Pereira, C., Soto, F. y De la Fuente, M. (2011). Carga de los cuidadores informales de personas con esquizofrenia: un desafío pendiente para la reforma de la atención en salud mental. Revista de Salud Pública, 15(2), 6-17. https://doi.org/10.31052/1853.1180.v15.n2.7020.

Avşar, U., Avşar, U.Z., Cansever, Z., Yucel, A., Cankaya, E., Certez, H., Keles, M., Aydinli, B. y Yucelf, N. (2015). Caregiver Burden, Anxiety, Depression, and Sleep Quality Differences in Caregivers of Hemodialysis Patients Compared With Renal Transplant Patients. Transplantation Proceedings, 47(5), 1388-1391. http://doi.org/10.1016/j.transproceed.2015.04.054.

Bardak, S., Demir, S., Aslan, E., Turgutalp, K., Celikcan, H. D., Dolarslan, M. E., Kilıcarslan, C., Karasu, F., Jini Gunes, A., Kurt, C. y Kiykım, A. (2018). The other side of the coin in renal replacement therapies: the burden on caregivers. International Urology and Nephrology, 51(2), 343-349. https://doi.org/10.1007/s11255-018-2029-0.

Bawazier, L.A., Stanley, I., Sianipar, W. y Suhardjono, S. (2018). Anxiety and depression among caregivers of hemodialysis patients at the Indonesian national referral hospital. Medical Journal of Indonesia, 27(4), 271-278. https://doi.org/10.13181/ mji.v27i4.2999.

Caputo, J., Pavalko, E.K., y Hardy, M.A. (2016). The Long-Term Effects of Caregiving on Women's Health and Mortality. Journal of Marriage and Family, 78(5), 1382-1398. https://doi.org/10.1111/jomf.12332.

Cervantes, L., Carr, A. L., Welles, C. C., Zoucha, J., Steiner, J. F., Johnson, T., Earnest, M., Camacho, C., Suresh, K. y Hasnain-Wynia, R. (2020). The Experience of Primary Caregivers of Undocumented Immigrants with End-Stage Kidney Disease that Rely on Emergency-Only Hemodialysis. Journal of General Internal Medicine, 35(8), 2389-2397. https:// doi.org/10.1007/s11606-020-05696-3.

Collins, L. y Swartz, K. (2011). Caregiver Care. American Academy of Family Physicians, 83(11), 1309-1317. https://www.aafp. org/afp/2011/0601/p1309.pdf.

Cubas, S.R, Sánchez, G.N. y Gálvez, D.N. (2019). Sobrecarga del cuidador del paciente con tratamiento de diálisis peritoneal. Enfermería Nefrológica, 22(3), 317-322. http://dx.doi.org/10.4321/S2254-28842019000300011.

Delgado, Q.E., Barajas, G.T., Uriostegui, E.L. y López, L.V. (2016). Sobrecarga y percepción de la calidad de vida relacionada con el cuidador primario del paciente hemodializado. Revista Cubana de Medicina General Integral, 32(4), 1-9. 
Denardi, C.E., Beuter, M., Brondani, C.M., Regina, P.M., Brentano, A.M. y Da Silva, C. (2013). A Diálise Peritoneal na vivência de familiares cuidadores. Revista da Rede de Enfermagem do Nordeste, 14(3), 541-548.

Díaz, N.K., Ramones, D.M., Vargas, E. y Alejo, M. (2018). Sobrecarga del cuidador familiar principal en pacientes pediátricos con enfermedad renal crónica según la escala de Zarit. Salud, Arte y Cuidado, 11(1), 33-44.

Einollahi, B., Taheri, S., Nemati, E., Abbaszadeh, S., Pourfarziani, V. y Nourbala, M. (2009). Burden among Care-givers of Kidney Transplant Recipients and its Associated Factors. Saudi Journal of Kidney Disease Transplant, 20(1), 30-34.

Figueroa L., A., Gonzalez B., M.A. y Alarcon I., J. (2016). Medical Expenditure for Chronic Diseases in Mexico: The Case of Selected Diagnoses Treated by the Largest Care Providers. Plos One, 11(1). https://doi.org/10.1371/journal.pone.0145177.

Franco, M.V. y Romero, M.E. (2019). Condiciones de cuidado en cuidadores de pacientes en diálisis peritoneal asociadas a sobrecarga. Ciencia E Innovación En Salud, 66, 1-12. https://doi.org/10.17081/innosa.66.

Haley, W.E., Roth, D.L., Howard, G. y Safford, M.M. (2010). Caregiving Strain and Estimated Risk for Stroke and Coronary Heart Disease Among Spouse Caregivers: Differential Effects by Race and Sex. Stroke, 41(2), 331-336. https://doi.org/10.1161/ STROKEAHA.109.568279.

Hoang, V.L., Green, T. y Bonner, A. (2018). Informal caregivers' experiences of caring for people receiving dialysis: A mixed methods systematic review. Journal of Renal Care, 44(2), 82-95. https://doi.org/10.1111/jorc.12235.

Hoenig, J. y Hamilton, M. (1966). The schizophrenic patient in the community and his effect on the household. International Journal of Social Psychiatry, 12(3), 165-176. https://doi.org/10.1177/002076406601200301.

Instituto Nacional de Estadística, Geografía e Informática (2019). Características de las defunciones registradas en México durante 2018. Comunicado de prensa núm. 538/19.

Kang, A., Yu, Z., Foo, M., Chan, C.M. y Griva, K. (2019). Evaluating Burden and Quality of Life among Caregivers of Patients Receiving Peritoneal Dialysis. Peritoneal Dialysis International, 39(2), 176-180. https://doi.org/10.3747/pdi.2018.00049.

Laguado J., E. (2019). Perfil del cuidador del paciente con Enfermedad Renal Crónica: una revisión de la literatura. Enfermería Nefrológica, 22(4), 352-359. http://dx.doi.org/10.4321/s2254-28842019000400002.

Lastiri, Q.H. (2016). Enfermedad renal crónica en México: una política nacional todavía pendiente. En J.A. Tamayo y O. y H.S. Lastiri Q. (Eds.): La enfermedad renal crónica en México. Hacia una política nacional para enfrentarla (pp. 1-16). Ciudad de México: Academia Nacional de Medicina.

López, E., Ávila, S., Reyes, A. y Miranda, I. (2018). Calidad de vida relacionada a la salud del cuidador primario del paciente con enfermedad renal crónica. Revista Médica del Colegio de Médicos y Cirujanos de Guatemala, 157(1). https://doi. org/10.36109/rmg.v157i1.82.

Menati, L., Torabi, Y., Andayeshgar, B. y Khatony, A. (2020). The Relationship Between Care Burden and Coping Strategies in Caregivers of Hemodialysis Patients in Kermanshah, Iran. Psychology Research and Behavior Management, 13, $133-140$. https://doi.org/10.2147/PRBM.S233103.

Mendoza, S.R., Hernández, G.E., Medina, P.M., Company, S.M., Gómez, P.M., Suárez, P.L., Navarro, V.F. y Fiuza, P.M. (2014). Perfil del cuidador principal en el área de salud de Gran Canaria. ENE, Revista de Enfermería, 8(2). http://dx.doi.org/10.4321/ S1988-348X2014000200002.

Montero, X., Jurado, S., Valencia, A., Méndez, J. y Mora, I. (2014). Escala de carga del cuidador de Zarit: evidencia de validez en México. Psicooncología, 11(1), 71-85. https://doi.org/10.5209/rev_PSIC.2014.v11.n1.44918.

National Kidney Foundation (2002). Clinical Practice Guidelines for Chronic Kidney Disease: Evaluation, Classification and Stratification. American Journal of Kidney Disease, 39, 1-266.

Organización Mundial de la Salud (1999). WHO Fact Sheet “Caregiving”. Geneva: WHO.

Pérez, T.A., González, G.M., López S., A.M., Sánchez, V.R. y Selgas, R. (2017). Evaluación de la dieta en pacientes con enfermedad renal crónica sin diálisis y su relación con el estado nutricional. Nutrición Hospitalaria, 34(6), 1399-1407. http:// dx.doi.org/10.20960/nh.960.

Rivero G., R., Segura G., E. y Juárez C., N.B. (2015). Sobrecarga del cuidador primario de pacientes en programa de hemodiálisis generada por el reingreso hospitalario. Diálisis y Trasplante, 36(2), 72-77. https://doi.org/10.1016/j.dialis.2015.02.001.

Romero, M.E., Bohórquez, M.C. y Castro, M.K. (2018). Calidad de vida y sobrecarga percibida por cuidadores familiares de pacientes con enfermedad renal crónica, Cartagena (Colombia). Archivos de Medicina (Col), 18(1), 1-13. https://doi. org/10.30554/archmed.18.1.2520.2018.

Schene, A.H., Tessler, R.C. y Gamache, G.M. (1994). Instruments measuring family on caregiver burden in severe mental illness. Social Psychiatry and Psychiatry Epidemiology, 29(5), 228-240. https://doi.org/10.1007/BF00796381.

Schulz, R. y Beach, S.R (1999). Caregiving as a Risk Factor for Mortality. The Caregiver Health Effects Study. Journal of the American Medical Association, 282(23), 2215-2219. https://doi.org/10.1001/jama.282.23.2215.

Smagula, S.F., Beach, S., Rosso, A.L., Newman, A.B. y Schulz, R. (2017). Brain Structural Markers and Caregiving Characteristics as Interacting Correlates of Caregiving Strain. The American Journal of Geriatric Psychiatry, 25(6), 582-591. https:// doi.org/10.1016/j.jagp.2017.02.015. 
Surendran, P., Venugopal, V., Dongre, A. y Paninjukunnath, R. (2018). Perceived challenges faced by family caregivers of chronic kidney disease patients and suggested solutions: A qualitative study. International Journal of Medical Science and Public Health, 7(6), 487-492. http://doi.org/10.5455/ijmsph.2018.0307819032018.

Tseliou, F., Rosato, M., Maguire, A., Wright, D. y O’Reilly, D. (2018). Variation of Caregiver Health and Mortality Risks by Age: A Census-Based Record Linkage Study. American Journal of Epidemiology, 187(7), 1401-1410. https://doi.org/10.1093/ aje/kwx384.

Usman Shah, H., Atif, I., Rashid, F., Babar, M.W., Arshad, F., Qamar, W., Khan, O.A. y Qadir, M. (2017). Assessment of caregiver burden of patients receiving dialysis treatment in Rawalpindi. Journal of the Pakistan Medical Association, 67(10), 14981501. https://jpma.org.pk/PdfDownload/8383.

Velázquez, Y. y Espín, A. (2014). Repercusión psicosocial y carga en el cuidador informal de personas con insuficiencia renal crónica terminal. Revista Cubana de Salud Pública, 40(1), 3-17.

Zarit, S.H., Eiler, J. y Hassinger, M.J. (1985). Clinical assessment. En J. E. Birren y K. W. Schaie (Eds.): Handbook of the psychology of aging (2nd ed.). New York: Van Nostrand Rinehold.

Zarit, S.H., Reever, K.E. y Bach-Peterson, J. (1980). Relatives of the impaired elderly: correlates of feelings of burden. The Gerontologist, 20(6), 649-655. https://doi.org/10.1093/geront/20.6.649. 